\title{
Mapeamento da precipitação mínima provável para o sul de Minas Gerais
}

\author{
Léo F. Ávila ${ }^{1}$, Carlos R. de Mello ${ }^{1} \&$ Marcelo R. Viola ${ }^{1}$
}

\section{RESUMO}

0 mapeamento da precipitação mínima provável associada a um período, local e freqüência de ocorrência, consiste em importante ferramenta para subsídios de práticas agrícolas. 0 objetivo neste trabalho foi mapear a precipitação provável mínima mensal e quinzenal para o Sul do Estado de M inas Gerais, por meio de técnicas geoestat́sticas. 0 nível de probabilidade considerado foi de $75 \%$ e o período analisado foi a estação chuvosa (outubro a março). 0 s valores de precipitação provável foram estimados pela distribuição de probabilidade Log-N ormal, após teste de sua adequação, por Kolmogorov-Smirnov, a partir de séries históricas de dados totais diários de precipitação referentes a 69 estações pluviométricas da região e arredores. Os model os de semivariograma exponencial e esférico ajustados pela máxima verossimilhança (MV) e por mínimos quadrados ponderados (MQP) foram modelados para a escolha do melhor modelo que representasse o semivariograma experimental. Com base no grau de dependência espacial e pela val idação cruzada, o modelo exponencial ajustado por M Q P apresentou melhor desempenho. Os mapas de precipitação provável mostraram considerável variabilidade durante a estação chuvosa, com os maiores valores estimados para as regiões das Serras da M antiqueira e Canastra.

Palavras-chave: geoestatística, continuidade espacial, planejamento da irrigação

\section{Probable minimum precipitation mapping for the Southern Minas Gerais, Brazil}

\begin{abstract}
The probable minimum precipitation associated to the period, location and frequency of occurrence represents an important tool to support agricultural practices. The objective of this work was to map the probable minimum monthly and fortnightly precipitation for Southern M inas Gerais State, applying geostatistical procedures. The probability level considered was $75 \%$ and the period evaluated was the rainy season. The values of probable minimum precipitation were estimated by Log-Normal Probability Distribution, following Kolmogorov-Smirnov adequacy test and using long-term daily precipitation data set of 69 pluviometric stations in the region and its surroundings. Exponential and spherical semi-variogram models, adjusted by Maximum Likelihood (ML) and M inimum W eighted Square (MWS), were modelled to choose the best to represent the experimental semi-variogram. Based on Spatial Dependence Degree and Cross-Validation, the exponential model adjusted by MWS presented better performance. The probable minimum precipitation maps have shown considerable variability during the rainy season. The greatest values were estimated for the region close to M antiqueira and Canastra Ranges.
\end{abstract}

Key words: geostatistic, spatial continuity, irrigation planning 


\section{INTRODUÇÃO}

A estimativa da precipitação com determinado nível de probabilidade é de suma importância para o planejamento agrícola, possibilitando a previsão da melhor época de preparo do solo, colheita, semeadura, aplicação de adubos e defensivos, e lâmina suplementar de irrigação.

O Sul de Minas Gerais é uma das regiões agropecuárias mais significativas do Brasil e o terceiro maior Produto Interno Bruto (PIB) do Estado de Minas Gerais. Destacam-se as produções de café de alto padrão, tanto para o mercado interno quanto para exportação de milho, para atender à demanda de várias fábricas de ração instaladas na região, as quais visam sustentar um dos maiores rebanhos leiteiros do País e, mais recentemente, tem-se destacado a fruticultura de clima temperado e a citricultura (Fundação João Pinheiro, 2004). Neste contexto, é salutar não só o desenvolvimento mas, também, o levantamento de informações técnicas que venham subsidiar o desenvolvimento econômico da região, especialmente associado ao mapeamento de variáveis climáticas fundamentais para a agricultura, como a precipitação provável, contribuindo para o desenvolvimento de uma agricultura eficiente e sustentável.

Precipitação provável é definida como sendo a precipitação pluviométrica mínima que apresenta probabilidade específica de ocorrência baseada em uma longa série histórica de dados (Hann, 2002), ou seja, é a lâmina mínima de chuva que se espera ocorrer, em dado período do ano e em determinado nível de probabilidade. Geralmente, este nível de probabilidade é de 75\%, para fins agrícolas (Bernardo et al., 2006); sua estimativa está associada a uma freqüência de ocorrência e é obtida mediante a utilização de modelos teóricos de probabilidades, ajustados a uma série histórica de dados representativa.

Existem diversas distribuições de probabilidade visando à estimativa da precipitação provável, cuja adoção é baseada na sua capacidade em estimar a freqüência de ocorrência em função de seus parâmetros de escala e de forma, medida através de testes de aderência; dentre essas distribuições se destacam: Distribuição Gama (Castro Neto \& Silveira, 1981a,b, 1983; Assis, 1993; Ribeiro \& Lunardi, 1997; Soares, 1999; Murta et al., 2005; Longo et al., 2006; Ribeiro et al., 2007; Silva et al., 2007; Sampaio et al., 2007) e Distribuição Log-Normal (Eltz et al., 1992; Sampaio et al., 1999; Junqueira Júnior et al., 2007; Ribeiro et al., 2007). Mello et al. (1994) descrevem a distribuição Log-Normal 2 parâmetros, como amplamente utilizada em hidrologia e hidroclimatologia, enfatizando que vários estudos constataram que esta distribuição pode ser adequada e recomendada para modelar probabilisticamente a precipitação, dependendo, no entanto, da escala temporal de sua ocorrência.

Um dos principais ramos de pesquisa em hidrologia e climatologia consiste, atualmente, na geração de mapas para subsidiar a implantação de projetos agrícolas e ambientais, por meio da análise de técnicas de interpolação espacial, reconstituindo a distribuição espacial da variável. Diversos estudos comparativos abrangendo métodos de interpolação aplicados a variáveis agroclimáticas, concluíram que o método geoestatístico se sobressai sobre os demais e deve ser aplicado quando possível (Mello et al., 2003a; Buytaert et al., 2006). Melo Júnior et al. (2006) estudando a distribuição espacial da frequiência de chuvas na região hidrográfica do Atlântico, no leste de Minas Gerais observaram, de modo geral, que o interpolador geoestatístico proporcionou boas estimativas dos totais médios anuais de chuva.

Desta forma, objetivou-se, neste trabalho, desenvolver o mapeamento da precipitação mínima provável mensal e quinzenal, ao longo do período chuvoso (outubro a março), na região sul de Minas Gerais, a nível de probabilidade de 75\%, com o emprego de técnicas geoestatísticas, para fornecer subsídios técnicos para implantação de projetos agrícolas na região.

\section{MATERIAL E MÉTODOS}

Foram utilizadas séries históricas, com no mínimo 15 anos de monitoramento, de precipitação diária anual, disponíveis

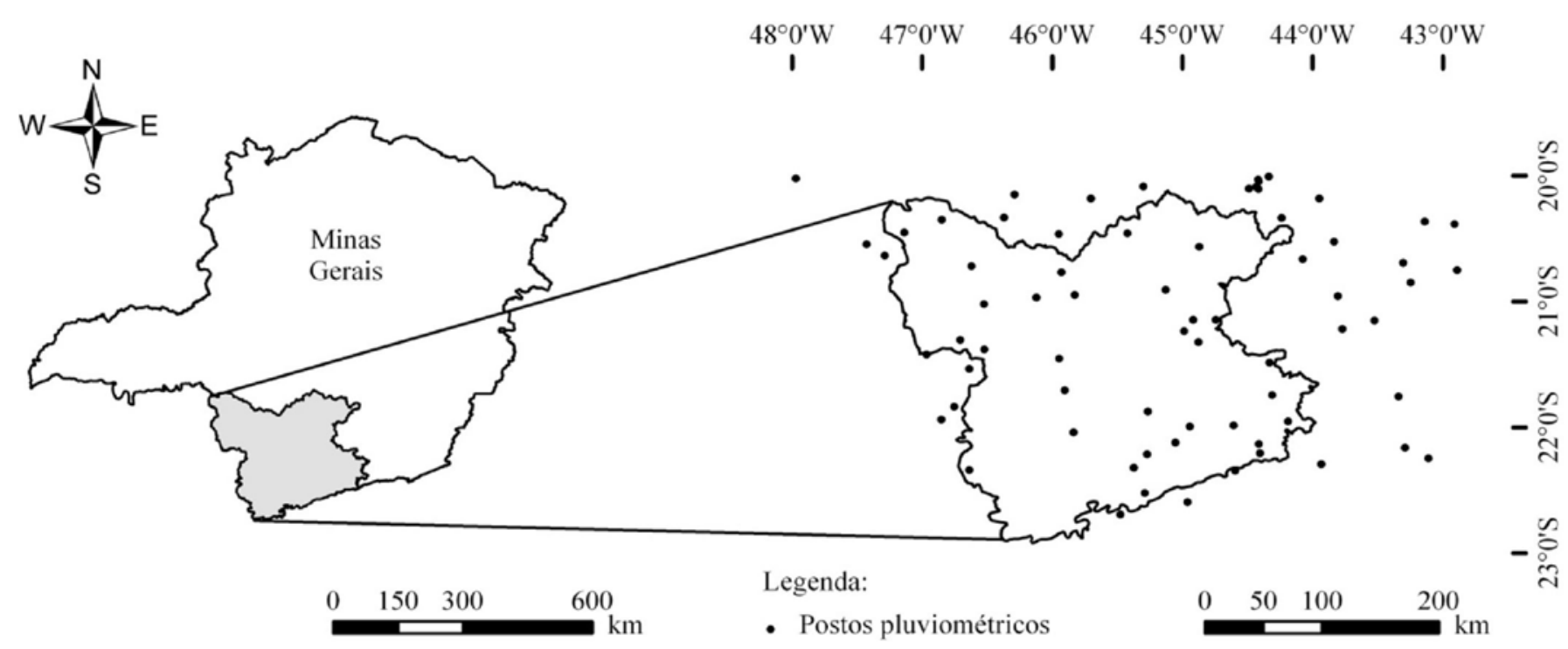

Figura 1. Estado de M inas Gerais, com destaque para a região Sul e postos pluviométricos utilizados 
no Sistema de Informações Hidrológicas da Agência Nacional de Águas (Hidroweb/ANA), para 69 postos pluviométricos localizados no Sul de Minas Gerais e arredores (Figura 1). Os dados diários de precipitação pluvial referentes aos meses de outubro a março, foram totalizados em períodos mensais e quinzenais, constituindo-se assim as respectivas séries históricas referentes a estas durações. Posteriormente, a distribuição Log-Normal (2 parâmetros) foi ajustada para estimativa da precipitação mínima provável associada a esses períodos, a nível de $75 \%$ de probabilidade de ocorrência. Com a finalidade de verificar a adequação dos dados à distribuição, aplicou-se o teste de Kolmogorov-Smirnov a nível de significância de 5\% de probabilidade (Longo et al., 2006).

Uma etapa fundamental da análise geoestatística consiste no ajuste do semivariograma teórico aos semivariogramas experimentais, realizado por métodos matemáticos, estimandose os parâmetros que estruturam tais modelos (efeito pepita, contribuição e alcance); desta forma, se testaram os modelos exponencial e esférico, pelos métodos de ajuste da máxima verossimilhança (MV) e de mínimos quadrados ponderados (MQP). Fez-se a análise da anisotropia do fenômeno por intermédio da construção dos semivariogramas experimentais, em várias direções, avaliando-se visualmente e removendo-a através de recursos oferecidos pelo software ArcGIS 9.2 Environmental Systems Research Institute, (ESRI, 2004). As direções adotadas para a respectiva análise foram $0^{\circ}, 45^{\circ}, 90^{\circ} \mathrm{e}$ $135^{\circ}$, consideradas convencionais na geoestatística (Isaaks \& Srivastava, 1989).

Para análise e escolha do modelo de semivariograma que melhor se adequou, avaliaram-se dois critérios: grau de dependência espacial (GD), calculado segundo Mello (2004), pela razão entre a variância estrutural $\left(\mathrm{C}_{1}\right)$ e o patamar $\left(\mathrm{C}_{0}+\mathrm{C}_{1}\right)$, o qual possui a seguinte classificação: $<25 \%$ (fraco), entre 25 e $75 \%$ (moderado), $>75 \%$ (forte); o segundo critério utilizado se baseia na técnica de validação cruzada ou auto-validação, que consiste em estimar, com base no modelo de semivariograma ajustado, os pontos amostrados possibilitando, assim, comparar o valor estimado ao real, trabalhando-se com o erro médio reduzido ( $\overline{\mathrm{ER}}$ ) e com o desvio padrão dos erros reduzidos $\left(\mathrm{S}_{\mathrm{ER}}\right)$; neste sentido, o melhor modelo é aquele que apresenta menor $\overline{\mathrm{ER}}$ e $\mathrm{S}_{\mathrm{ER}}$ mais próximo de 1 (Mello, 2004). Em todas as análises estatísticas, incluindo-se a análise exploratória dos dados como gráficos de tendência, histogramas de freqüência das diferenças entre pares de pontos e identificação de dados discrepantes (“outliers") através de gráficos "box plot", utilizou-se o software R, com o pacote geoR (Ribeiro Júnior \& Diggle, 2001), ambos de livre acesso e de acordo com a licença GPL (General Public Licence). Definido o modelo de semivariograma que melhor se ajustou, prosseguiu-se com a fase de geração de mapas por meio do interpolador geoestatístico (krigagem) na espacialização dos dados de precipitação provável, fase em que foi utilizada a extensão ArcMAP, do software ArcGIS 9.2 (ESRI, 2004), trabalhando-se com os parâmetros do modelo de semivariograma escolhido.

\section{RESULTADOS E DISCUSSÃO}

\section{Análise da modelagem dos semivariogramas}

Pelo teste de adequação estatística de Kolmogorov-Smirnov, verificou-se que das 1242 distribuições ajustadas, ou seja, 69 séries históricas divididas em 12 períodos quinzenais e 6 mensais, apenas 4 não se adequaram à distribuição Log-Normal (2 Parâmetros). Este resultado vem confirmar estudos prévios que testaram e recomendaram esta distribuição ao estudo da precipitação provável (Junqueira Júnior et al., 2007; Longo et al., 2006; Mello et al., 1994).

Pode-se verificar, na Figura 2, os principais gráficos oriundos da análise exploratória dos dados para precipitação provável mínima mensal do mês de dezembro, ressaltando-se comportamento aproximadamente semelhante aos dos demais eventos, em termos de tendência e de normalidade. $\mathrm{O}$ gráfico box plot (2A) permite detectar dados discrepantes (outliers) que possam influenciar substancialmente o comportamento dos semivariogramas experimentais, sobretudo na sua parte inicial (Ribeiro Júnior \& Diggle, 2001), removendo-os para posterior análise espacial. Os gráficos de tendência $(2 \mathrm{~B}, 2 \mathrm{C})$ apresentam distribuição dos dados nas direções Norte-Sul (Latitude) e Leste-Oeste (Longitude), não se verificando tendências aí, a medida por que se caminham nessas direções, fato que atende ao princípio de não tendenciosidade, o qual é fundamental em geoestatística para a aplicação da hipótese intrínseca (Mello, 2004). O histograma de freqüência das diferenças entre pares de pontos amostrados (Figura 2D) indica a presença de normalidade multivariada devido à boa distribuição da freqüência, em termos de normalidade das
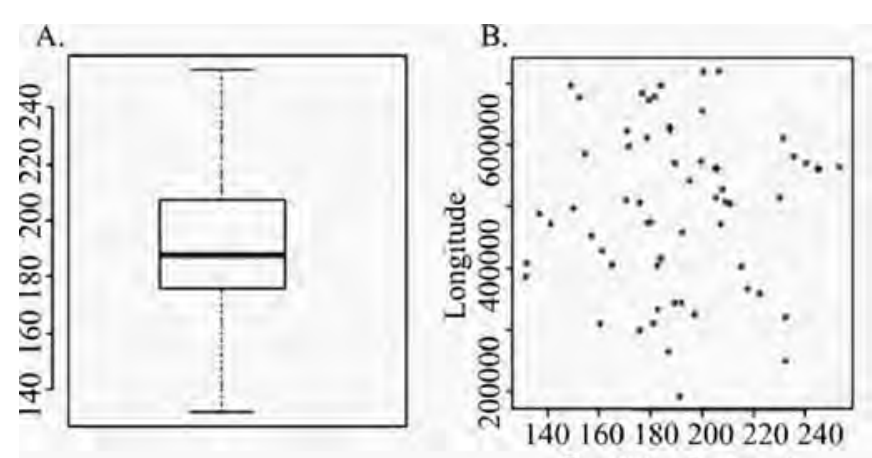

Precipitação $(\mathrm{mm})$
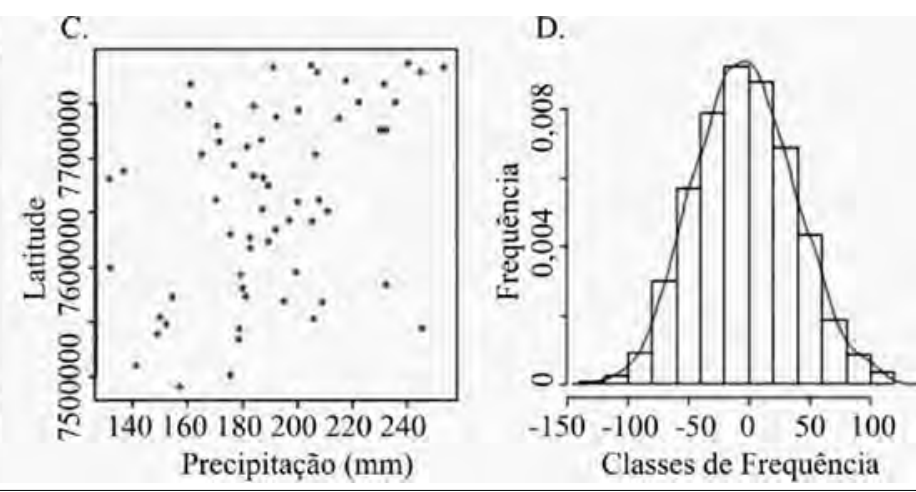

Figura 2. Gráficos "Box plot" $(A)$, tendência $(B, C)$ e histograma das diferenças entre pares de pontos (D), para precipitação provável mensal do mês de dezembro 
Tabela 1. Parâmetros dos modelos de semivariograma, grau de dependência espacial (GD), erro médio reduzido ( $\overline{\mathrm{ER}})$ e desvio padrão dos erros reduzidos $\left(S_{E R}\right)$ para o período mensal

\begin{tabular}{|c|c|c|c|c|c|c|c|c|}
\hline Mês & Modelo & Método & $\mathrm{CO}$ & $\mathrm{Cl}+\mathrm{CO}$ & $A(\mathrm{~km})$ & GD (\%) & $\overline{E_{R}}$ & $\mathrm{~S}_{\mathrm{ER}}$ \\
\hline \multirow{4}{*}{ Outubro } & Exponencial & MV & 131,32 & 409,22 & 270 & 68 & 0,00 & 0,25 \\
\hline & Esférico & MV & 131,54 & 353,04 & 270 & 63 & 0,00 & 0,24 \\
\hline & Exponencial* & MQP & 106,22 & 449,78 & 270 & 76 & 0,00 & 0,26 \\
\hline & Esférico & MQP & 107,85 & 364,93 & 306 & 70 & 0,00 & 0,25 \\
\hline \multirow{4}{*}{ Novembro } & Exponencial & MV & 464,80 & 1168,20 & 30 & 60 & $-0,01$ & 1,04 \\
\hline & Esférico & MV & 631,98 & 1354,28 & 300 & 53 & $-0,01$ & 1,05 \\
\hline & Exponencial* & MQP & 549,53 & 1477,32 & 300 & 63 & $-0,01$ & 1,04 \\
\hline & Esférico & MQP & 546,77 & 1319,39 & 300 & 59 & $-0,01$ & 1,05 \\
\hline \multirow{4}{*}{ Dezembro } & Exponencial & MV & 548,90 & 1757,50 & 300 & 69 & $-0,01$ & 0,49 \\
\hline & Esférico & MV & 565,10 & 1505,70 & 300 & 62 & $-0,01$ & 0,49 \\
\hline & Exponencial* & MQP & 479,98 & 2158,84 & 300 & 78 & $-0,01$ & 0,51 \\
\hline & Esférico & MQP & 499,03 & 1659,40 & 300 & 70 & $-0,01$ & 0,50 \\
\hline \multirow{3}{*}{ J aneiro } & Exponencial & MV & 990,50 & 2118,50 & 250 & 53 & 0,00 & 0,49 \\
\hline & Esférico & MV & 977,80 & 2068,10 & 250 & 53 & 0,00 & 0,48 \\
\hline & Exponencial* & MQP & 933,11 & 2195,52 & 300 & 57 & 0,00 & 0,49 \\
\hline \multirow{3}{*}{ Fevereiro } & Exponencial & MV & 473,21 & 1432,40 & 300 & 67 & 0,00 & 0,49 \\
\hline & Esférico & MV & 472,27 & 1256,12 & 300 & 62 & 0,00 & 0,50 \\
\hline & Esférico* & MQP & 373,70 & 1764,27 & 679 & 79 & 0,00 & 0,50 \\
\hline \multirow{4}{*}{ Março } & Exponencial & MV & 265,77 & 698,79 & 250 & 62 & 0,00 & 0,35 \\
\hline & Esférico & MV & 264,38 & 648,59 & 250 & 59 & 0,00 & 0,32 \\
\hline & Exponencial* & MQP & 215,13 & 705,85 & 250 & 70 & 0,00 & 0,36 \\
\hline & Esférico & $M Q P$ & 212,78 & 590,94 & 250 & 64 & 0,00 & 0,36 \\
\hline
\end{tabular}

* modelos de semivariogramas escolhidos para o mapeamento da precipitação provável

diferenças, considerada condição fundamental em estudos geoestatísticos, sobretudo quando se utilizam algoritmos de máxima verossimilhança, a qual consiste da aplicação de um Modelo Multivariado Normal (Ribeiro Júnior \& Diggle, 2001).

Apresentam-se, nas Tabelas 1, 2, e 3, os resultados da autovalidação (validação cruzada), grau de dependência espacial e parâmetros dos semivariogramas ajustados pelas metodo- logias empregadas. Em virtude da semelhança da magnitude dos erros médios reduzidos e do desvio-padrão dos erros médios na maioria dos casos, o critério decisivo de escolha do melhor modelo a ser utilizado na krigagem foi o que apresentou maior grau de dependência espacial, com exceção para a segunda quinzena de janeiro, quando os modelos ajustados se apresentaram GD muito próximos e elevados (Tabela

Tabela 2. Parâmetros dos modelos de semivariogramas, grau de dependência espacial (GD), erro médio reduzido ( $\overline{\text { ER }}$ ) e desvio padrão dos erros reduzidos $\left(S_{E R}\right)$ para a 1 à quinzena

\begin{tabular}{ccccccccc}
\hline Mês & Modelo & Método & C0 & C1 + C0 & A (km) & GD (\%) & $\mathbf{E}_{\mathbf{R}}$ & $\mathbf{S}_{\text {ER }}$ \\
& Exponencial* & MV & 29,49 & 73,09 & 150 & 60 & 0,00 & 0,10 \\
Outubro & Esférico & MV & 30,62 & 72,56 & 300 & 58 & 0,00 & 0,10 \\
& Exponencial & MQP & 36,25 & 72,50 & 150 & 50 & $-0,04$ & 0,12 \\
& Esférico & MQP & 36,25 & 72,50 & 150 & 50 & 0,00 & 0,10 \\
\hline \multirow{4}{*}{ Novembro } & Exponencial & MV & 135,32 & 283,27 & 300 & 52 & 0,00 & 0,17 \\
& Esférico & MV & 135,10 & 279,56 & 300 & 52 & 0,00 & 0,17 \\
& Exponencial* & MQP & 112,52 & 286,57 & 300 & 61 & 0,00 & 0,17 \\
& Esférico & MQP & 115,03 & 263,06 & 300 & 56 & 0,00 & 0,17 \\
\hline \multirow{2}{*}{ Dezembro } & Esférico & MV & 240,96 & 526,90 & 73 & 54 & 0,00 & 0,27 \\
& Exponencial* & MQP & 234,04 & 15405,80 & 445 & 98 & 0,00 & 0,28 \\
\hline \multirow{3}{*}{ Janeiro } & Exponencial & MV & 354,95 & 936,83 & 350 & 62 & $-0,01$ & 0,29 \\
& Esférico & MV & 360,80 & 852,43 & 350 & 58 & 0,00 & 0,29 \\
& Exponencia* & MQP & 344,93 & 945,40 & 350 & 64 & $-0,01$ & 0,29 \\
\hline \multirow{2}{*}{ Fevereiro } & Exponencial & MV & 143,51 & 535,14 & 200 & 73 & $-0,01$ & 0,30 \\
& Esférico* & MV & 140,53 & 462,77 & 200 & 70 & $-0,01$ & 0,30 \\
& Exponencial & MQP & 174,54 & 522,18 & 200 & 67 & $-0,01$ & 0,30 \\
\hline \multirow{2}{*}{ Março } & Exponencial* & MV & 338,60 & 947,08 & 240 & 64 & 0,00 & 0,34 \\
& Esférico & MV & 346,60 & 850,70 & 240 & 59 & 0,00 & 0,34 \\
& Exponencial & MQP & 354,30 & 930,25 & 240 & 62 & 0,00 & 0,34 \\
\hline
\end{tabular}

* modelos de semivariogramas escolhidos para o mapeamento da precipitação provável 
Tabela 3. Parâmetros dos modelos dos semivariogramas, grau de dependência espacial (GD), erro médio reduzido ( $\overline{\mathrm{ER}}$ ) e desvio padrão dos erros reduzidos $\left(S_{E R}\right)$, para a 2a quinzena

\begin{tabular}{|c|c|c|c|c|c|c|c|c|}
\hline Mês & Modelo & Método & $\mathrm{CO}$ & $\mathrm{Cl}+\mathrm{CO}$ & $A(\mathrm{~km})$ & GD (\%) & $E_{R}$ & $\mathbf{S}_{\mathrm{ER}}$ \\
\hline \multirow{3}{*}{ Outubro } & Exponencial & MV & 118,44 & 254,89 & 500 & 54 & 0,00 & 0,17 \\
\hline & Esférico & MV & 117,25 & 247,34 & 500 & 53 & 0,00 & 0,17 \\
\hline & Exponencial* & MQP & 74,81 & 321,65 & 500 & 77 & 0,00 & 0,18 \\
\hline \multirow{3}{*}{ Novembro } & Exponencial* & MV & 99,50 & 340,15 & 100 & 71 & $-0,01$ & 0,24 \\
\hline & Esférico & MV & 80,97 & 281,24 & 100 & 71 & $-0,01$ & 0,24 \\
\hline & Esférico & MQP & 95,55 & 316,99 & 100 & 70 & $-0,01$ & 0,24 \\
\hline \multirow{4}{*}{ Dezembro } & Exponencial & MV & 311,02 & 833,42 & 100 & 63 & $-0,01$ & 0,36 \\
\hline & Esférico & MV & 243,27 & 705,92 & 100 & 66 & $-2,57$ & 0,78 \\
\hline & Exponencial* & $M Q P$ & 97,62 & 672,81 & 37 & 85 & $-0,02$ & 0,38 \\
\hline & Esférico & MQP & 143,11 & 671,62 & 82 & 79 & $-0,02$ & 0,38 \\
\hline \multirow{4}{*}{ J aneiro } & Exponencial & MV & 55,28 & 562,31 & 50 & 90 & $-0,02$ & 0,35 \\
\hline & Esférico & MV & 23,40 & 437,04 & 50 & 95 & $-1,24$ & 0,52 \\
\hline & Exponencial & $M Q P$ & 0,00 & 878,89 & 84 & 100 & $-0,03$ & 0,36 \\
\hline & Esférico* & $M Q P$ & 0,00 & 452,07 & 72 & 100 & $-0,02$ & 0,35 \\
\hline \multirow{3}{*}{ Fevereiro } & Exponencial & MV & 187,24 & 542,29 & 50 & 65 & $-0,01$ & 0,29 \\
\hline & Esférico & MV & 133,76 & 480,53 & 50 & 72 & $-0,02$ & 0,30 \\
\hline & Esférico* & $M Q P$ & 122,17 & 420,90 & 39 & 71 & $-0,01$ & 0,29 \\
\hline \multirow{4}{*}{ Março } & Exponencial & MV & 6,81 & 397,42 & 100 & 98 & $-0,04$ & 0,27 \\
\hline & Esférico & MV & 6,46 & 224,62 & 90 & 97 & $-0,01$ & 0,24 \\
\hline & Exponencial* & $M Q P$ & 0,00 & 205,75 & 227 & 100 & $-0,03$ & 0,24 \\
\hline & Esférico & MQP & 0,00 & 198,64 & 57 & 100 & $-0,04$ & 0,25 \\
\hline
\end{tabular}

* modelos de semivariogramas escolhidos para o mapeamento da precipitação provável

3), sendo a escolha do melhor modelo determinada a partir de $\overline{\mathrm{ER}}$ e $\mathrm{S}_{\mathrm{ER}}$.

Houve melhor ajuste do modelo exponencial pelo método MQP em 55\% dos casos; situação semelhante foi constatada no trabalho de Mello et al. (2008), em estudo sobre modelagem de semivariogramas, para chuvas intensas, com diferentes durações e tempos de retorno, no Estado de Minas Gerais, os quais se ajustaram os modelos esférico, exponencial e gaussiano e constataram superioridade do modelo exponencial, em $75 \%$ das situações analisadas. Neste mesmo aspecto, Buytaert et al. (2006) e Mello et al. (2003b), estudaram a variabilidade espacial da precipitação, respectivamente, na região dos Andes Equatoriais e no Estado de Minas Gerais, com o emprego do modelo exponencial para o mapeamento dessa variável e encontraram forte estrutura de dependência espacial do modelo exponencial, recomendando-o para estudos ligados à distribuição espacial das chuvas.

As Figuras 3 e 4 apresentam os semivariogramas escolhidos em cada situação destacada nas Tabelas 1, 2 e 3, sendo possível observar bom ajuste dos semivariogramas ajustados aos semivariogramas experimentais.

Os alcances médios obtidos para os períodos considerados foram de $350 \mathrm{~km}$ para a precipitação mínima provável mensal e de 280 e $163 \mathrm{~km}$, respectivamente para as $1^{\text {a }}$ e $2^{\text {a }}$ quinzenas. A máxima distância geográfica verificada dentro do Sul de Minas Gerais é de aproximadamente $450 \mathrm{~km}$ no sentido leste-oeste. O comportamento dos alcances mostra influência mútua em praticamente toda a região, significando que a distribuição espacial da precipitação provável no Sul de Minas é regida pelos mesmos mecanismos físicos. De fato, praticamente não se verifica variabilidade espacial importante em termos climáticos na região, oscilando entre Cwa e Cwb, pela classificação de Köppen, e entre Úmido e Superúmido por Thortwaite (Dantas et al., 2007). Por se tratar de totais precipitados acumulados no mês ou em quinzenas, os eventos de precipitação de origem convectiva, comuns no verão, são pouco expressivos em termos de total precipitado, não exercendo influência significativa na distribuição espacial das chuvas; entretanto, eventos de precipitação oriundos de sistemas frontais (frentes frias) são altamente expressivos na região no período chuvoso e responsáveis pela grande maioria dos totais precipitados e no número de dias chuvosos; fisicamente, são eventos que afetam, de forma simultânea, grandes áreas, significando que, no caso do Sul de Minas Gerais, a presença de uma frente fria afetará toda a região em curto espaço de tempo, fazendo com que o comportamento das precipitações acumuladas seja homogêneo.

\section{Mapeamento da precipitação provável mínima noSul de Minas Gerais}

Nas Figuras 5, 6 e 7 se encontram os mapas de precipitação provável mínima mensal e 1a e 2a quinzenas, respectivamente, para o Sul de Minas Gerais, a partir dos semivariogramas escolhidos, com remoção da anisotropia. Houve grande variabilidade das lâminas estimadas para os meses analisados compreendidos no período chuvoso; observa-se também concentração dos maiores valores da precipitação mínima provável mensal na porção Sudeste da região, atingindo valores de até $253 \mathrm{~mm}$ em dezembro. O mês de outubro apresentou as menores lâminas, com valores variando entre 34 e $104 \mathrm{~mm}$, notando-se intensidades maiores de precipitação na parte sul da região, com comportamento similar em uma faixa que se estende no sentido leste-oeste. 

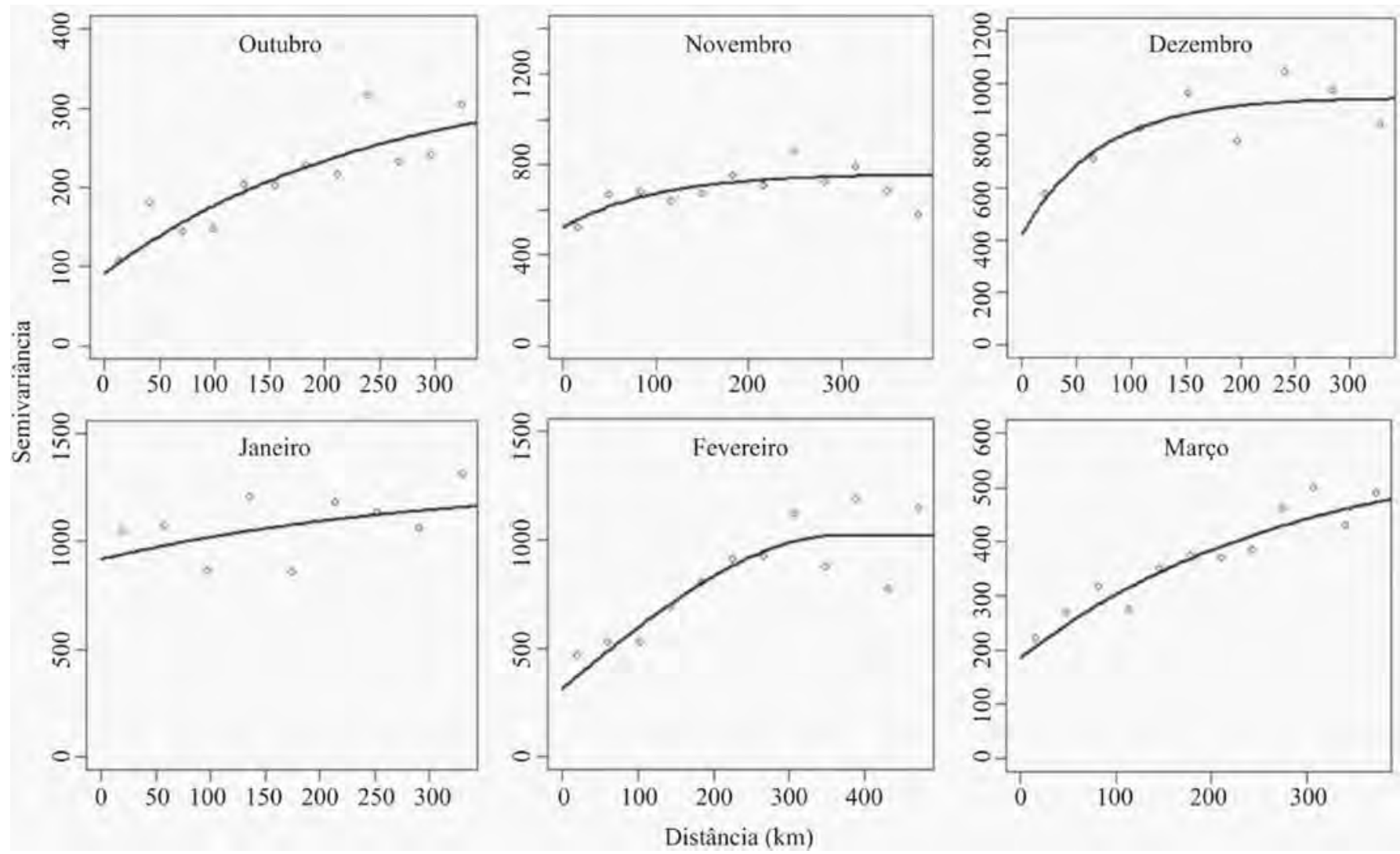

Figura 3. Semivariogramas teóricos escolhidos para o mapeamento da precipitação provável mínima no Sul de M inas Gerais, para o período mensal

Em termos de variabilidade dos valores encontrados, os meses de outubro e fevereiro apresentaram as maiores diferenças proporcionais entre os valores extremos (o limite superior é, aproximadamente, 3 vezes maior que o limite inferior para o mês de outubro e de 4 vezes para o mês de fevereiro). A explicação se baseia no fato de que o mês de outubro é o primeiro do período chuvoso e, conforme Mello et al. (2007), representa a transição entre os dois períodos característicos da região, os períodos seco e o chuvoso, apresentando valores de precipitação, naturalmente, oscilantes devido à influência periódica do fenômeno El Niño, que afeta o regime de chuvas no Sudeste do Brasil a cada 5 anos (Moreira, 1999); além disso, a porção sul da região é atingida com maior freqüência por frentes frias, especialmente no inverno e na transição para a primavera, como em setembro e outubro, produzindo dias mais chuvosos no entorno da Serra da Mantiqueira.

Analisando-se as Figuras 6 e 7, relativas ao período quinzenal, é possível constatar alta variabilidade dos dados em todos os meses estudados, em especial, na 1a quinzena; os meses de outubro, janeiro e março, apresentaram variabilidade superior a 17 vezes entre os limites superior e inferior, especialmente janeiro e março, na $1^{\mathrm{a}}$ quinzena; na $2^{\mathrm{a}}$ quinzena se observaram altas oscilações; contudo, inferiores às constatadas para a $1^{\text {a }}$ quinzena, cujo comportamento demonstra ser possível dias chuvosos menos expressivos na 1a quinzena dos meses do período chuvoso em algumas partes da região, o que gera maior variabilidade espacial.

Os maiores valores de precipitação prevaleceram nas proximidades das Serras da Canastra (noroeste da região) e Mantiqueira (sul e sudeste da região), especialmente na última, fato explicado pela influência orográfica nessas áreas, provocando precipitações mais freqüentes, fruto da presença de massas de ar mais úmidas. No tocante à implantação de culturas irrigadas, na região próxima à Serra da Mantiqueira pode-se prever, com razoável nível de segurança, uma lâmina natural mais expressiva, em particular no plantio, período que se inicia em outubro e se estende até dezembro e, em alguns casos específicos, até janeiro.

Observa-se predomínio dos maiores valores próximos à Serra da Mantiqueira e, em alguns casos, junto à Serra da Canastra, na sua vertente sul (Figuras 6 e 7), o que justifica a influência orográfica das serras na ocorrência das precipitações, como constatado por Mello et al. (2007), que conduziram estudo sobre estiagem no Estado de Minas Gerais e concluíram sobre a relevante influência da topografia no favorecimento de precipitações nas vertentes de sistemas como Espinhaço, Mantiqueira e Canastra. As precipitações quinzenais máximas encontradas foram 123 e $141 \mathrm{~mm}$ para as $1^{\mathrm{a}}$ e $2^{\mathrm{a}}$ quinzenas, respectivamente, ao longo da Serra da Mantiqueira, mas é necessário comentar que, normalmente, os solos destas áreas são pouco profundos e, portanto, com baixa capacidade de armazenamento de água, como Cambissolos e Neossolos Litólicos (Araújo, 2006). Avalia-se, desta forma, que apesar da constatação das maiores lâminas de precipitação mínima provável, essas áreas necessitarão, inevitavelmente, de um suplemento adicional de água via irrigação, para o desenvolvimento produtivo de algumas culturas. 

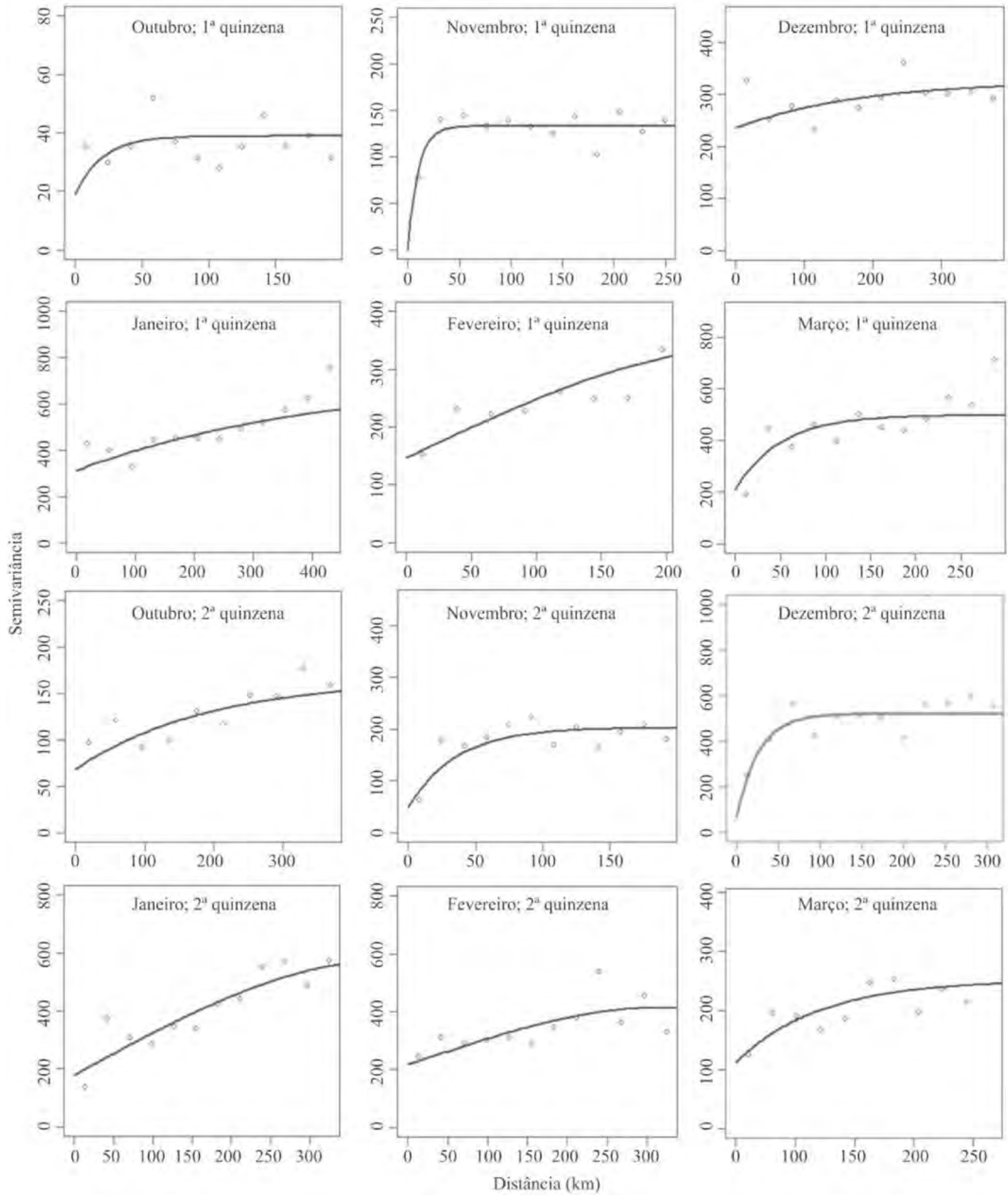

Figura 4. Semivariogramas teóricos escolhidos para o mapeamento da precipitação provável mínima no Sul de Minas Gerais, para as 1aㅡ e 2ª quinzenas

Os mapas produzidos demonstram homogeneidade na porção sul da região, predominantemente com os maiores totais precipitados. Esta porção da região é caracterizada por uma agricultura desenvolvida, com aplicação de irrigação suplementar para culturas como laranja, hortaliças e fruticul- tura de clima temperado. Neste contexto, os resultados gerados serão empregados no sentido de se promover dimensionamentos dos sistemas de irrigação, de forma mais sustentável, tal como no preparo do solo, com redução da compactação e melhoria de sua capacidade produtiva, especialmente a preci- 

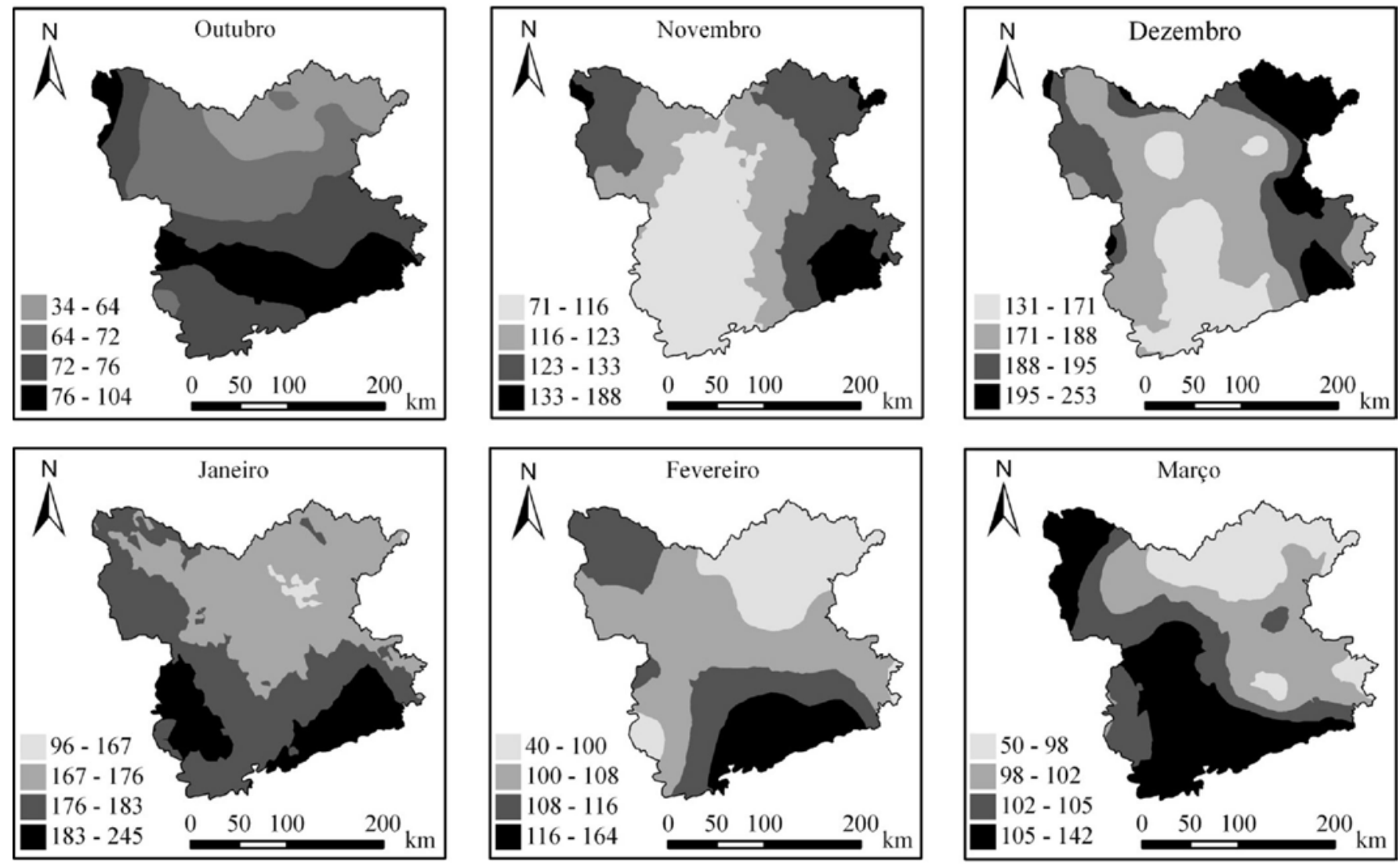

Figura 5. M apas de precipitação provável mínima (mensal), em mm, para o Sul de M inas Gerais
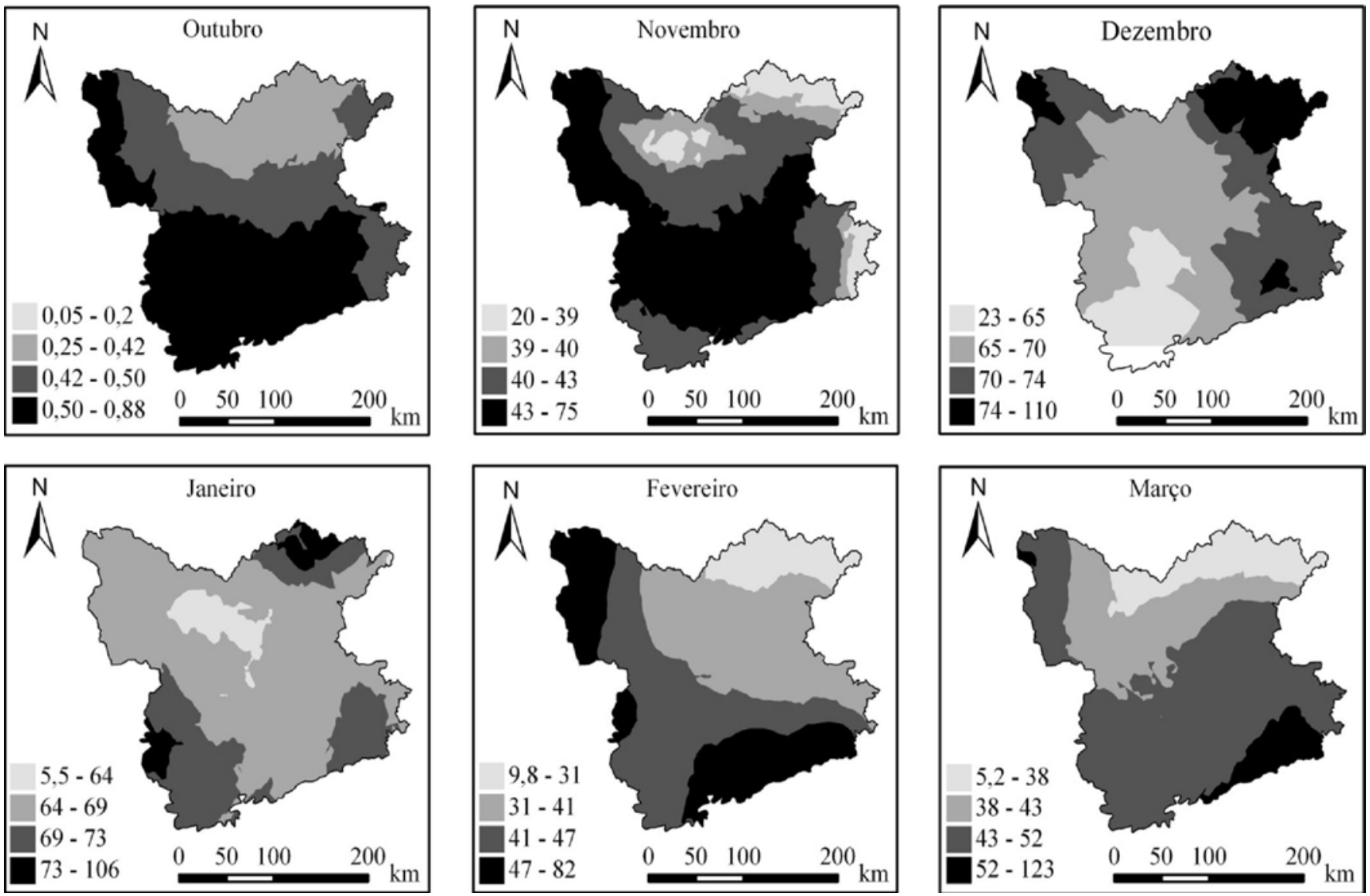

Figura 6. Mapas de precipitação provável mínima (1a quinzena), em mm, para o Sul de Minas Gerais 

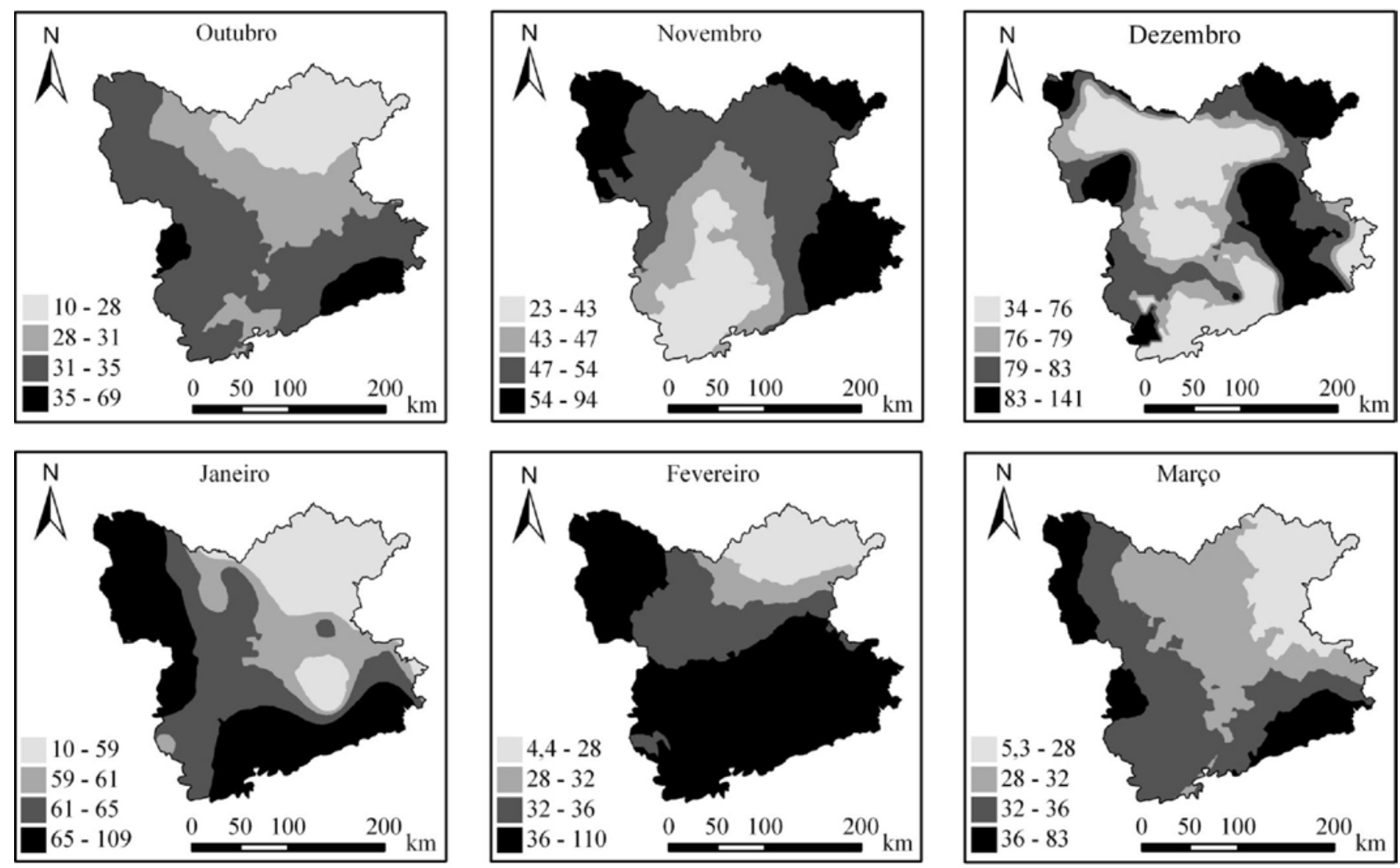

Figura 7. Mapas de precipitação provável mínima (2ª quinzena), em $\mathrm{mm}$, para o Sul de Minas Gerais

pitação mínima provável quinzenal, a qual fornece maiores subsídios para os agricultores e produtores, de maneira geral.

\section{CONCLUSÕES}

1. Verificou-se considerável variabilidade da precipitação provável mínima mensal na região, ao longo do período chuvoso, com valores variando entre 34 e $253 \mathrm{~mm}$.

2. Os meses que apresentaram os maiores valores para as séries mensais foram dezembro e janeiro com, respectivamente, 253 e $245 \mathrm{~mm}$.

3. Os valores mais expressivos de precipitação provável mínima foram predominantemente na porção sul.

4. Os resultados obtidos podem ser usados no subsídio a agricultores e produtores, fornecendo informações que auxiliam na tomada de decisão da época de preparo do solo e semeadura, e no dimensionamento de sistemas de irrigação.

\section{LITERATURA CITADA}

Araújo, A. R. Solos da bacia do Alto Rio Grande, MG: Base para estudos hidrológicos e aptidão agrícola. Lavras: UFLA, 2006. 345p. Tese Doutorado

Assis, F. N. Ajuste da função gama aos totais semanais de chuva de Pelotas, RS. Revista Brasileira de Agrometeorologia, v.1, n.1, p.131-136, 1993.
Bernardo, S.; Soares, A. A.; Mantovani, E. C. Manual de irrigação. 8.ed. Viçosa: Imprensa Universitária. 2006. 625p.

Buytaert, W.; Celleri, R.; Willems, P.; Bièvre, B. de; Wyseure, G. Spatial and temporal rainfall variability in mountainous areas: a case study from the South Ecuadorian Andes. Journal of Hydrology, v.329, p.413-421, 2006.

Castro Neto, P.; Silveira, J. V. Precipitação provável para Lavras, região Sul de Minas Gerais, baseada na função de distribuição de probabilidade gama I: Períodos mensais. Ciência e Prática, v.5, n.2, p.144-151, 1981a.

Castro Neto, P.; Silveira, J.V. Precipitação provável para Lavras, região Sul de Minas Gerais, baseada na função de distribuição de probabilidade gama II: Períodos de quinze dias. Ciência e Prática, v.5, n.2, p.152-162, 1981b.

Dantas, A. A. A.; Carvalho, L. G.; Ferreira, E. Classificação e tendências climáticas em Lavras, MG. Ciência e Agrotecnologia, v.31, n.6, p.1862-1866, 2007.

Eltz, F. L. F.; Reichert, J. M.; Cassol, E. A. Período de retorno de chuvas em Santa Maria, RS. Revista Brasileira de Ciência do Solo, v.16, n.2, p.265-269, 1992.

ESRI - Environmental Systems Research Institute. ArcGIS 9: Getting started with ArcGIS. Redlands:ESRI, 2004. 265p.

Fundação João Pinheiro, MG. Perfil de Minas Gerais, 8.ed. Belo Horizonte: FJP, 2004. 352p.

Haan, C. T. Statistical Methods in Hydrology. 2.ed. Ames: The Iowa State University Press, 2002. 377p.

Isaaks, E. H.; Srivastava, R. M. An Introduction to Applied Geostatistics. New York: Oxford University, 1989. 600p. 
Junqueira Júnior, J. A.; Gomes, N. M.; Mello, C. R. de; Silva, A. M. da. Precipitação provável para a região de Madre de Deus, Alto Rio Grande: modelos de probabilidades e valores característicos. Ciência e Agrotecnologia, v.31, n.3, p.842850, 2007.

Longo, A. J.; Sampaio, S. C.; Queiroz, M. M. F.; Sussek, M. Uso das distribuições gama e log-normal na estimativa de precipitação provável quinzenal. Varia Scientia, v.6, n.11, p.107-118, 2006.

Mello, C. R. de; Lima, J. M.; Silva, A. M.; Mello, J. M.; Silva, M. S. Krigagem e inverso do quadrado da distância para interpolação dos parâmetros da equação de chuvas intensas. Revista Brasileira de Ciência do Solo, v.27, n.5, p.925-933, 2003 a.

Mello, C. R. de; Sá, M. A. C.; Curi, N.; Mello, J. M.; Viola, M. R.; Silva, A. M. Erosividade mensal e anual no Estado de Minas Gerais. Pesquisa Agropecuária Brasileira, v.42, n.4, p.537545, 2007.

Mello, C. R. de; Silva, A. M.; Lima, J. M.; Ferreira, D.F.; Oliveira, M.S. Modelos matemáticos para predição da chuva de projeto para regiões do estado de Minas Gerais. Revista Brasileira de Engenharia Agrícola e Ambiental, v.7, n.1, p.121-128, 2003b.

Mello, C. R. de; Viola, M. R.; Mello, J. M.; Silva, A. M. Continuidade espacial de chuvas intensas no estado de Minas Gerais. Ciência e Agrotecnologia, v.32, n.2, p. 532-539, 2008.

Mello, J. M. de. Geoestatística aplicada ao inventário florestal. Piracicaba: ESALQ, 2004. 110p. Tese Doutorado

Mello, M. H. A.; Arruda, H. V.; Ortolani, A. A. Probabilidade de ocorrência de totais pluviais máximos horários, em Campinas, São Paulo. Revista do Instituto Geológico, v.15, n.1/2, p.5967, 1994.

Melo Júnior, J. C. F de; Sediyama, G. C.; Ferreira, P. A.; Leal, B. G.; Minusi, R. B. Distribuição espacial da freqüência de chuvas na região hidrográfica do Atlântico, Leste de Minas Gerais. Revista Brasileira de Engenharia Agrícola e Ambiental, v.10, n.2, p.417-425, 2006.
Moreira, A. A. M. A influência da circulação de macroescala sobre o clima de Belo Horizonte: estudo sobre as possíveis influências do fenômeno El Niño sobre o clima local. Belo Horizonte: UFMG, 1999. 186p. Dissertação Mestrado

Murta, R. M.; Teodoro, S. M; Bonomo, P.; Chaves, A. C. Precipitação pluvial mensal em níveis de probabilidade pela distribuição gama para duas localidades do sudoeste da Bahia. Ciência e Agrotecnologia, v.29, p.988-994, 2005.

Ribeiro, A. M. de A.; Lunardi, C. A precipitação mensal provável para Londrina, PR, através da função gama. Energia na Agricultura, v.12, p.37-44, 1997.

Ribeiro, B. T.; Avanzi, J. C; Mello, C. R. de; Lima, J. M. de; Silva, M. L. N. Comparação de distribuições de probabilidade e estimativa da precipitação provável para a região de Barbacena, MG. Ciência e Agrotecnologia, v.31, n.5, p.1297-1302, 2007.

Ribeiro Júnior, P. J.; Diggle, P. P. GeoR: A package for geoestatistical analysis. R - News, v.21, n.2, p.15-18, 2001.

Sampaio, S. C.; Corrêa, M. M.; Souza, M. R.; Guimarães, J. C.; Silva, A. M. Precipitação provável para município de LavrasMG, utilizando a distribuição log-normal. Ciência e Agrotecnologia, v.23, n.2, p.382-389, 1999.

Sampaio, S. C.; Queiroz, M. M. F. de; Frigo, E.; Longo, A. J.; Suszek, M. Estimativa e distribuição de precipitações decendiais para o Estado do Paraná. Irriga, v.12, n.1, p.38-53, 2007.

Silva, J. C. da; Heldwein, A. B.; Martins, F. B.; Trentin, G.; Grimm, E. L. Análise de distribuição de chuva para Santa Maria, RS. Revista Brasileira de Engenharia Agrícola e Ambiental, v.11, n.1, p.67-72, 2007.

Soares, A. A. Evapotranspiração de referência e precipitação provável no Estado de Minas Gerais visando à elaboração de projetos de irrigação. Revista Engenharia Agrícola, v.18, n.4, p.14-18, 1999. 